\title{
On P-nipotence of Finite Groups
}

\author{
Shitian Liu \\ College of Science, Sichuan University of Science \& Engineering \\ Zigong 643000, China \\ E-mail: liust@suse.edu.cn
}

\begin{abstract}
A subgroup $\mathrm{H}$ is said to be weakly $c^{*}$-normal in a group $\mathrm{G}$ if there exists a subnormal subgroup $\mathrm{K}$ of $\mathrm{G}$ such that $H K=G$ and $H \cap K$ is s-quasinormally embedded in G. We give some results which generalize some authors' results.
\end{abstract}

Keywords: Weakly $c^{*}$-normality, p-nilpotence, s-quasinormally embedded

\section{Introduction}

In this paper the word group is always finite. Ore $(1937$, p150) gives quasinormality of subgroups. A subgroup $\mathrm{H}$ is said to be quasinormal in $\mathrm{G}$ if for every subgroup $\mathrm{K}$ of $\mathrm{G}$ such that $H K=K H$. A subgroup $\mathrm{H}$ of a group $\mathrm{G}$ is said to be s-quasinormal in $\mathrm{G}$ if $\mathrm{H}$ permutes with every Sylow subgroup of $\mathrm{G}$. This concept was introduced by Kegel (1962, p 205), and extensively studied (Deskins, 1963, p126-131). Ballester-Bolinches and PedrazaAguilera (1998, p114) introduce the conception of s-quasinormally embedded in G if for each prime divisor $\mathrm{p}$ of $\mathrm{H}$, a Sylow p-subgroup of $\mathrm{H}$ is also a Sylow p-subgroup of some s-quasinormal subgroup of $\mathrm{G}$. Wei and Wang (2007, p212)introduced the notion of $c^{*}$-normality, a subgroup $\mathrm{H}$ of $\mathrm{G}$ is said to be $c^{*}$-normal in $\mathrm{G}$ if there exists a subgroup $K \leq G$ such that $G=H K$ and $H \cap K$ is s-quasinormally embedded in G.

For some notions and notations, the reader is referred to Robinson (1995)and Huppert (1968).

\section{Some definitions and preliminary results}

A subgroup $\mathrm{H}$ is called weakly c-normal in a group $\mathrm{G}$ if there exists a subnormal subgroup $\mathrm{T}$ of $\mathrm{G}$ such that $G=H T$ and $H \cap T \leq H_{G}$, where $H_{G}$ is the largest normal subgroup of $\mathrm{G}$ contained in $\mathrm{H}$. The conception of weakly c-normality was introduced by Lu, Guo, and Shum (2002, p 5506).

Definition 2.1 A subgroup $\mathrm{H}$ is said to be weakly $c^{*}$-normal in $\mathrm{G}$ if there exists a subnormal subgroup $\mathrm{T}$ of $\mathrm{G}$ such that $G=H T$ and $H \cap T \leq H_{s} G$, where $H_{s} G$ is s-quasinormally embedded subgroup of $\mathrm{G}$ contained in $\mathrm{H}$.

Lemma 2.1 (Ballester-Bolinches and Pedraza-Aguilera, 1998, Lemma 1) Suppose that U is s-quasinormally embedded in a group $\mathrm{G}$, and that $H \leq G$ and $K \triangleleft G$.

(1) If $U \leq H$, then $\mathrm{U}$ is s-quasinormally embedded in $\mathrm{H}$.

(2) If $\mathrm{UK}$ is s-quasinormally embedded in $\mathrm{G}$, then $U K / K$ is s-quasiormally embedded in $G / K$.

(3) If $K \triangleleft H$ and $H / K$ is s-quasinormally embedded in $G / K$, then $\mathrm{H}$ is s-quasinormally embedded in $\mathrm{G}$.

Lemma 2.2 Let $\mathrm{G}$ be a group. Then the following statements hold.

(1) If $\mathrm{H}$ is weakly $C^{*}$-normal in $\mathrm{G}$ and $H \leq M \leq G$, then $\mathrm{H}$ is weakly $c^{*}$-normal in $\mathrm{M}$.

(2) Let $N \triangleleft G$ and $N \leq H$. Then $\mathrm{H}$ is weakly $c^{*}$-normal in $\mathrm{G}$ if and only if $H / N$ is weakly $c^{*}$-normal in $G / N$.

(3)Let $\pi$ be a set of primes. $\mathrm{H}$ is a $\pi$-subgroup of $\mathrm{G}$ and $\mathrm{N}$ a normal $\pi^{\prime}$-subgroup of $\mathrm{G}$, if $\mathrm{H}$ is weakly $c^{*}$-normal in $\mathrm{G}$, then $\mathrm{HN} / \mathrm{N}$ is weakly $c^{*}$-normal in $G / N$.

(4)Let $L \leq G$ and $H \leq \Phi(L)$ If $\mathrm{H}$ is weakly $c^{*}$-normal in $\mathrm{G}$, then $\mathrm{H}$ is s-quasinormally embedded in $\mathrm{G}$. 
(5)Let $\mathrm{H}$ is $c^{*}$-normal in $\mathrm{G}$, then $\mathrm{H}$ is weakly $c^{*}$-normal in $\mathrm{G}$.

Proof. (1) If $\mathrm{H}$ is weakly $c^{*}$-normal in $\mathrm{G}$, that is, there exists a subnormal subgroup $\mathrm{T}$ of $\mathrm{G}$ such that $H T=G$ and $H \cap T$ is s-quasinormally embedded in $\mathrm{G}$, then $M=M \cap G=(M \cap T) H$. Since $\mathrm{T}$ is subnormal in $\mathrm{G}$, then $M \cap T$ is subnormal in $\mathrm{M}$, and $H \cap(M \cap T)$ is s-quasinormally embedded in $\mathrm{M}$. So we have $\mathrm{H}$ is weakly $c^{*}$-normal in $\mathrm{M}$.

(2) If $\mathrm{H}$ is weakly $c^{*}$-normal in $\mathrm{G}$, then there exists a subnormal $\mathrm{T}$ of $\mathrm{G}$ such that $G=H T$ and $H \cap T$ is s-quasinormally embedded in $\mathrm{G}$. Then $G / N=(H / N)(T N / N)$, where $T N / N$ is subnormal in $G / N$ and $(H / N) \cap$ $(T N / N)$ is s-quasinormally embedded in $G / N$. Then $H / N$ is weakly $c^{*}$-normal in $G / N$. The converse part can be proved similarly.

(3) If $\mathrm{H}$ is weakly $c^{*}$-normal in $\mathrm{G}$, then there exists a subnormal subgroup $\mathrm{T}$ of $\mathrm{G}$ such that $G=H T$ and $H \cap T$ is s-quasinormally embedded in G. Since $|G|_{\pi^{\prime}}=|T|_{\pi^{\prime}}=|T N|_{\pi^{\prime}}$, then $N \leq T$. Clearly $(H N / N)(T / N)=G / N$ and $(H N / N) \cap(T / N)=(H \cap T) N / N$ is s-quasinormally embedded in $G / N$.

(4) Since $\mathrm{H}$ is weakly $c^{*}$-normal in $\mathrm{G}$, then there exists a subnormal subgroup $\mathrm{T}$ such that $G=H T$ and $H \cap T$ is s-quasinormally embedded in $G \cdot L=L \cap(H T)=H(T \cap L)$. Since $H \leq \Phi(L)$, then $L=T \cap L$ and so $L \leq T$, then $T=G$ and $H=H \cap T$ is s-quasinormally embedded in $\mathrm{G}$.

(5) The result is obvious.

Lemma 2.3 Let $\mathrm{M}$ be a maximal subgroup of $\mathrm{G}$ and $\mathrm{P}$ a normal Sylow p-subgroup of $\mathrm{G}$ such that $G=P M$, where $\mathrm{p}$ is a prime, then $P \cap M$ is a normal subgroup of $\mathrm{G}$.

Lemma 2.4 (Wei and Wang, 2007, Lemma 2.5) Let G be a group, K an s-quasinormal subgroup of G, P a Sylow p-subgroup of $\mathrm{K}$ where $\mathrm{p}$ is a prime divisor of $|G|$. If either $P \leq O_{P}(G)$ or $K_{G}=1$, then $\mathrm{P}$ is s-quasinormal in $\mathrm{G}$.

Lemma 2.5 (Li, Wang and Wei, 2003, Lemma 2.2) Let $\mathrm{G}$ be a group and $\mathrm{P}$ is s-quasinormal p-subgroup of $\mathrm{G}$ where $\mathrm{p}$ is a prime, then $O_{P}(G) \leq N_{G}(P)$.

Lemma 2.6 (Wei and Wang, 2007, Lemma 2.8) Let $\mathrm{G}$ be a group and p a prime dividing $|G|$ with $(|G|, p-1)=1$.

(1) If $\mathrm{N}$ is normal in $\mathrm{G}$ of order $\mathrm{p}$, then $\mathrm{N}$ is in $Z(G)$.

(2) If G has cyclic Sylow p-subgroups, then $\mathrm{G}$ is p-nilpotent.

(3)If $M \leq G$ and $|G: M|=p$, then $M \triangleleft G$.

Lemma 2.7 (Huppert, 1968, IV, 5.4) Suppose that G is a group which is not p-nilpotent but whose proper subgroups are all p-nilpotent. Then $\mathrm{G}$ is a group which is not nilpotent but whose proper subgroups are all nilpotent.

Lemma 2.8 (Robinson, 1995, III, 5.2) Suppose that G is a group which is not nilpotent but whose proper subgroups are all nilpotent. Then

(1) $\mathrm{G}$ has a normal Sylow p-subgroup for some prime $\mathrm{p}$ and $G=P Q$, where $\mathrm{Q}$ is a non-normal cyclic qsubgroup for some prime $q \neq p$.

(2) $P / \Phi(P)$ is a minimal normal subgroup of $G / \Phi(P)$.

(3) If $\mathrm{P}$ is non-abelian and $p \neq 2$, then $\exp (P)=p$.

(4) If $\mathrm{P}$ is non-abelian and $p=2$, then $\exp (P)=4$.

(5)If $\mathrm{P}$ is abelian, then $\exp (P)=p$.

Lemma 2.9 Let $\mathrm{H}$ be a subgroup of $\mathrm{G}$. Then $\mathrm{H}$ is weakly $c^{*}$-normal in $\mathrm{G}$ if and only if there exists a subgroup $\mathrm{K}$ such that $G=H K$ and $H \cap K=H_{s G}$.

Proof. $\Leftarrow$ It is clear.

$\Rightarrow$ By definition 2.1, there exists a subnormal subgroup $\mathrm{L}$ of $\mathrm{G}$ such that $G=H L$ and $H \cap L \leq H_{s G}$ If $H \cap L<H_{s G}$, note that $K=L H_{s G}$, then $H K=H L H_{s G}=L H H_{S G}=L H=G$ and hence $H \cap K=H \cap L H H_{s G}=$ $(H \cap L) H_{s G}=H_{s G}$. 


\section{Main results}

Theorem 3.1 Let $\mathrm{G}$ be a group, $\mathrm{P}$ a Sylow $\mathrm{p}$-subgroup of $\mathrm{G}$, where $\mathrm{p}$ is a prime divisor of $|G|$ with $(|G|, p-1)=1$. If all maximal subgroups of $\mathrm{P}$ are weakly $c^{*}$-normal in $\mathrm{G}$, then $\mathrm{G}$ is p-nilpotent.

Proof. Suppose that the result is false, then we chose a minimal order $\mathrm{G}$ as a counterexample. We will prove by the following steps:

Steps 1 . For every proper subgroup of $\mathrm{G}$ is p-nilpotent, thus $\mathrm{G}$ is a group which is not p-nilpotent but whose proper subgroups are all p-nilpotent.

Let $\mathrm{M}$ be a maximal subgroup of $\mathrm{G}$, Then $P \cap M$ is a maximal p-subgroup of $\mathrm{P}$. By hypothesis, $P \cap M$ is weakly $c^{*}$-normal in $\mathrm{G}$ and so $P \cap M$ is weakly $c^{*}$-normal in $\mathrm{M}$ by lemma 2.2(1). Thus $\mathrm{M}, P \cap M$ satisfies the hypotheses of the theorem, the minimal choice of $\mathrm{G}$ implies that $\mathrm{M}$ is p-nilpotent. Then we have that $\mathrm{G}$ is not p-nilpotent but all proper subgroups are p-nilpotent. Then, by lemma 2.7 and lemma 2.8(1), G has a normal Sylow p-subgroup for some prime $\mathrm{p}$ and $G=P Q$, where $\mathrm{Q}$ is a non-normal cyclic q-subgroup for some prime $q \neq p$.

Steps 2. Let $\mathrm{L}$ be a minimal normal subgroup of $\mathrm{G}$ contained in $\mathrm{P}$, then $G / L$ is p-nilpotent, $\mathrm{L}$ is the unique minimal normal of $\mathrm{G}$ and $L \not \leq \Phi(G)$.

Since $P / L$ is a Sylow p-subgroup of $G / L$, we have $M / L$ is a maximal subgroup in $P / L$, where $\mathrm{M}$ is a maximal subgroup of P. Since M is weakly $c^{*}$-normal in G, by lemma $2.2(2) M / L$ is weakly $c^{*}$-normal in $G / L$. Thus $G / L, P / L$ satisfies the hypotheses of the theorem and so we have $G / L$ is p-nilpotent by the minimal choice of G. If $L_{1}$ is an another minimal normal subgroup, then $G / 1 \cong G / L \times G / L_{1}$ is p-nilpotent and so $L$ is unique. If $L \leq \Phi(G)$, then $G / \Phi(G)$ is p-nilpotent, and so is $\mathrm{G}$, a contradiction.

Steps 3. $\Phi(P) \neq 1$.

If $\Phi(P)=1$, then $\mathrm{P}$ is abelian. By steps 1 and lemma 2.8(5), $\exp (P)=p$. If $|P / \Phi(P)|=P^{n}$ and $P / \Phi(P)=<$ $x_{1} \Phi(P), x_{2} \Phi(P), \cdots, x_{n} \Phi(P)>$, then $P=<x_{1}, x_{2} \cdots, x_{n}>$. So we have $\left|<x_{1}>\right|=p$, and $<x_{i}>$ char $\mathrm{P}$, where $\mathrm{i}$ is nature number. And since $\mathrm{P}$ is normal in $\mathrm{G}$, then $\left\langle x_{i}>\right.$ are normal p-subgroup of $\mathrm{G}$ of order $\mathrm{p}$. Thus by lemma 2.6(1), we have $<x_{i}>\leq Z(G)$ for all $i=1,2, \cdots, n$, then $P \leq Z(G)$, then $\mathrm{G}$ is p-nilpotent, a contradiction. Thus $\Phi(P) \neq 1$.

Steps 4. $\mathrm{L}$ is a Sylow p-subgroup of $\mathrm{G}$.

By steps 3, $\Phi(P) \neq 1$, then $L \leq P$. If $L<P$, then for a maximal subgroup $\mathrm{M}$ of $\mathrm{P}, \mathrm{M}$ is weakly $c^{*}$-normal in $\mathrm{G}$ and so there exists a subnormal subgroup $\mathrm{K}$ such that $G=M K$ and $M \cap K$ is s-quasinormally embedded in $\mathrm{G}$. We consider the following cases.

1) $M \cap K=1$.

Since $|K|_{p}=|G: M|_{p}=|P Q: M Q|_{p}=|P: M|=p$, then $\mathrm{K}$ has a normal p-complement $Q_{1}$ which is also a Sylow q-subgroup of G. By Sylow theorem, there exists an element $g \in G \backslash Q$ such that $Q_{1}^{g}=Q$. Since $M \triangleleft P$, then $G=M K=(M K)^{g}=M K^{g}$. Since $K^{g} \cong K$ and $Q=Q_{1}^{g} \leq K^{g}$, this implies $K^{g} \leq N_{G}(Q)$ in this case $\mathrm{Q}$ is not normal in G. So we have $G=M K=(M K)^{g}=M N_{G}(Q)$. So we have $M \cap N_{G}(Q)=1$ and $N_{G}(Q) \leq K^{g}$. Thus $K^{g}=N_{G}\left(Q^{g}\right)=N_{G}(Q)$. If $\mathrm{H}$ be a sylow subgroup of $N_{G}(Q)$, then $K=H Q$ and $H P=P H=P$. This implies that $\mathrm{H}$ is s-quasinormal in $\mathrm{G}$, then by lemma 2.5 we have $O_{p}(H) \leq N_{G}(H)$, and $\mathrm{H}$ is normal in $\mathrm{G}$. Then $L=H \leq M$ since the minimality of $\mathrm{L}$ and $\mathrm{L}$ is unique. But $L \not \leq M$, a contradiction.

2) $M \cap K=M=M_{S G}$.

Then we have $M \leq K$, then $G=K$ is p-nilpotent, a contradiction.

3) $1<M_{s G}<M$.

Let $S=M \cap K$. Then $\mathrm{S}$ is s?quasinormally embedded in $\mathrm{G}$. Thus there exists an s-quasinormal subgroup $\mathrm{R}$ such that $\mathrm{S}$ is a Sylow p-subgroup of R. Then by lemma 2.5, we have $O^{p}(G) \leq N_{G}(S)$ and so $\mathrm{S}$ is normal in G, then we have, $S=P$ or $S=L$. If $S=P$. On the other hand, $|M|<|P|$, a contradiction. Then $S=L$ is a minimal normal Sylow p-subgroup of some s-quasinormal subgroup R of $\mathrm{G}$, then for any sylow q-subgroup $\mathrm{Q}$, we have $R Q=Q R$ is a subgroup of $\mathrm{G}$ and, if $Q S<G, Q \triangleleft R Q$ by (1), and so $L Q=L \times Q$. By steps 1 and Burnside's theorem, we have $\mathrm{G}$ is solvable. Thus $Q \leq C_{G}(L) \leq L$, a contradiction. Then $Q S=G$, then 
$G=P Q=Q S$ and so $P=S^{g}$ for some $g \in Q$, a contradiction.

Steps 5. Conclusions.

By steps $4, L=P$ is a Sylow p-subgroup of $\mathrm{G}$, then, by hypothesis, maximal subgroup $\mathrm{M}$ of $L=P$ is weakly -normal in $\mathrm{G}$. Then by lemma 2.10 there exists a subnormal subgroup $\mathrm{K}$ of $\mathrm{G}$ such that $G=M K$ and $M \cap K \leq M_{s G}$. Since $M_{s G}<L=P<L=P$, then $M_{S G}=1$ and $L Q / L$ is p-nilpotent since $G / L$ is p-nilpotent by steps 2, where Q is a Hall p'-subgroup of $\mathrm{G}$, then $L Q / L \triangleleft G / L$ and so $L Q \triangleleft G$. It follows from $\mathrm{Q} \operatorname{char} L Q \triangleleft G$ that $\mathrm{Q}$ is normal in $\mathrm{G}$. Therefore $\mathrm{G}$ is p-nilpotent.

Corollary 3.1 (Wei and Wang, 2007, Theorem 3.1) Let G be a group, P a Sylow p-subgroup of G, where p is a prime divisor of $|G|$ with $(|G|, p-1)=1$. If all maximal subgroups of $\mathrm{P}$ are $c^{*}$-normal in $\mathrm{G}$, then $\mathrm{G}$ is p-nilpotent.

Theorem 3.2 Let $\mathrm{G}$ be a group, $\mathrm{P}$ a Sylow p-subgroup of $\mathrm{G}$, where $\mathrm{p}$ is a prime divisor of $|G|$ with $(|G|, p-1)=1$. If all cyclic subgroups of $\mathrm{P}$ of order $\mathrm{p}$ or 4 (if $\mathrm{p}=2$ ) are weakly $c^{*}$-normal in $\mathrm{G}$, then $\mathrm{G}$ is $\mathrm{p}$-nilpotent.

Proof. Suppose that the result is false, then we chose a minimal order $\mathrm{G}$ as a counterexample. We will prove by the following steps:

Steps 1. Let $M$ be a proper subgroup of $G$, then $M$ is p-nilpotent. So $G$ is not p-nilpotent but all proper subgroups are p-nilpotent. Thus $G=P Q$, where $\mathrm{P}$ is a normal Sylow p-subgroup of $\mathrm{G}$ and $\mathrm{Q}$ is a non-normal cyclic Sylow q-subgroup of G. And so by Burnside's theorem $\mathrm{G}$ is solvable. Then $M \cap P$ is a Sylow p-subgroup of M. By hypothesis, for every cyclic subgroup of $P$ of order $p$ or 4 (if $p=2$ ) is weakly -normal in $G$, then By lemma 2.2(1), for every cyclic subgroup of $\mathrm{P} M$ of order $\mathrm{p}$ or 4 (if $\mathrm{p}=2$ ) is weakly $c^{*}$-normal in $\mathrm{M}$. Then $\mathrm{M}$, $M \cap P$ satisfies the hypotheses of the theorem, $\mathrm{M}$ is p-nilpotent by the minimal choice of $\mathrm{G}$, so we have: $\mathrm{G}$ is not p-nilpotent but all proper subgroups are p-nilpotent and so by lemma 2.7 and lemma 2.8(1), $G=P Q$, where $\mathrm{P}$ is a normal Sylow p-subgroup of $\mathrm{G}$ and $\mathrm{Q}$ is a non-normal cyclic Sylow q-subgroup of G.

Steps 2. Let $\mathrm{L}$ be a minimal normal subgroup of $\mathrm{G}$ contained in $\mathrm{P}$, then $\mathrm{L}$ is unique minimal normal p-subgroup for some prime of $|G|, G / L$ is p-nilpotent and $L \not \leq \Phi(G)$. Furthermore, $L=F(G)=C_{G}(L)$.

Since all cyclic subgroups of $\mathrm{P}$ of order $\mathrm{p}$ or $4\left(\right.$ if $\mathrm{p}=2$ ) is weakly $c^{*}$-normal in $\mathrm{G}$, then by lemma 2.2(2) all cyclic subgroups of $\mathrm{P} / \mathrm{L}$ with order $\mathrm{p}$ or 4 (if $\mathrm{p}=2$ ) is weakly $c^{*}$-normal in $G / L$, then the minimal choice of $\mathrm{G}$ implies that $G / L$ is p-nilpotent. If $L \leq \Phi(G)$, then $G / \Phi(G)$ is p-nilpotent and $\mathrm{G}$ is p-nilpotent, a contradiction. By lemma 2.6 (Li, etc, 2003), $F(G)=L$. By steps 1 , solubility of $\mathrm{G}$ implies that $L \leq C_{G}(F(G)) \leq F(G)$ and so $C_{G}(L)=F(G)=L$ as $\mathrm{L}$ is abelian.

Steps 3. Conclusions.

By steps $2 C_{G}(L)=F(G)=L$. But on the other hand, for $x \in P,<x>$ is weakly $c^{*}$-normal in $\mathrm{G}$, then there exists a subnormal subgroup $\mathrm{T}$ of $\mathrm{G}$ such that $G=\langle x\rangle T$ and $\langle x\rangle \cap T$ is s-quasinormally embedded in $\mathrm{G}$. By lemma 2.7 and lemma 2.8, we have if $\mathrm{p}$ is odd or $\mathrm{P}$ is abelian, then $\exp (P)=p$ or if $p=2 \exp (P)=4$. Since $F(G)=<x_{1}, x_{2}, \cdots, x_{n}>=L,\left|<x_{i}>\right|=p$ or 4 and $<x_{i}>$ char $\mathrm{P}$ since $\mathrm{P}$ is normal in $\mathrm{G}$. Thus $F(G)=L=<x_{i}>$. So we have $L Q=Q L=L \times Q$, Then $Q \leq N_{G}(L)$, then $G=P \times Q$ is nilpotent, a contradiction.

Corollary 3.2 (Li and Wang, 2004, Theorem 4.1) Suppose $\mathrm{G}$ is a group, $\mathrm{p}$ is a fixed prime number. If every element of $P_{p}(G)$ is contained in $Z_{\infty}(G)$. If $p=2$, every cyclic subgroup of order 4 of $\mathrm{G}$ is s-quasinormal in $\mathrm{G}$, then $\mathrm{G}$ is p-nilpotent.

\section{References}

Ballester-Bolinches, A., Pedraza-Aguilera, M. C. (1998). Sufficient conditions for supersolubility of finite groups, J. Pure Appl.Algebra, 127, 113-138.

Deskins, W. E. (1963). On quasinormal subgroups of finite groups, Math. Z, 82, 125-132.

Huppert, B. (1968). Endlinche Gruppen. I. New York: Springer-Varlag.chapter IV.

Kegel, O. (1962). Sylow-gruppen und subnormalteiler endlicher gruppen, Math. Z, 78, 205-22.

Li, Y. Wang, Y., Wei, H. (2003). The influence of s-quasinormality of some subgroups of a finite group, Arch. 
Math, 81, 245-252.

Li, Y. Wang, Y. (2004). On s-quasinormally embedded subgroups of finite group, J. Algebra, 281, 109-123.

Ore, O. (1937). Structures and group theory I, Duke Math. J, 3, 149-174.

Robinson, D. J. S(1995). A course in theory of groups 2nd. New York: Springer-Varlag.

Wei, H., Wang, Y. (2007). On $c^{*}$-normality and its properties. J. Group Theory, 10, 211-223.

Zhu, L., Guo, W., Shum, K. P. (2002). Weakly c-normal subgroups of finite groups and their properties, Comm. Algebra, 30(11), 5505-5512. 